\title{
Traffic design to reduce the number of OEO conversions in multi-stage access network
}

\author{
Tatsuya Shimada ${ }^{\text {a) }}$, Hideaki Kimura, and Hisaya Hadama \\ NTT Access Network Service Systems Laboratories, NTT Corporation \\ 1-1, HIkarinooka, Yokosuka-shi, Kanagawa 239-0847, Japan \\ a)shimada.tatsuya@lab.ntt.co.jp
}

\begin{abstract}
It is important to realize the efficient accommodation of access traffic for various services that will have a wide dynamic range of bandwidth. To achieve this, we are studying a new access network based on multi-stage semiconductor optical amplifiers (SOAs), which has a multi-access point that separately accommodates different services. This paper describes the configuration and advantage of this network and presents a traffic design obtained by simulations that examined the number of optical-electrical-optical (OEO) conversions at first-in first-out (FIFO) controlling packets with a view to reducing
\end{abstract} power consumption.

Keywords: access network, SOA, FIFO, OEO, power consumption Classification: Fiber-optic communication

\section{References}

[1] Special Feature: M. Jinno, H. Kimura, Y. Hibino, K. Uehara, N. Kukutsu, F. Ito, and S. Matsuoka, "Towards Ultrahigh-speed High-capacity Networks," NTT Technical Journal, vol. 7, no. 5, pp. 1-7, May 2009.

[2] H. Hadama, A. Tsutsui, N. Takahashi, and A. Takahara, "Design Concept for Future Carrier Networks," MD 1.2 LEOS Summer Topicals, 2009.

[3] S. J. Ben Yoo, "Optical Packet and Burst Switching Technologies for the Future Photonic Internet," J. Lightw. Technol., vol. 24, no. 12, pp. 44684492, Dec. 2006.

[4] S. Narikawa, H. Sanjoh, N. Sakurai, and K. Kumozaki, "Gbit-Class Transmission Using SOA Data Rewriter for WDM-PON," IEICE Trans. Commun., vol. E91-B, no. 2, pp. 399-408, Feb. 2008.

[5] L. Chiaraviglio, M. Mellia, and F. Neri, "Energy-aware Backbone Networks: A Case Study," First Int. Workshop on Green Communications (GreenComm'09), Dresden, June 2009.

\section{Introduction}

Various activities have been reported in relation to future networks in response to the diverse requirements of users and services $[1,2]$. One require- 
ment for future access networks is that they support a wide dynamic range of bandwidth such as a huge bandwidth service (HBS), a broadband packet service (BPS) and a tiny bandwidth mass service (TMS) [2]. Therefore, it is important to consider the efficient accommodation of access traffic. If we accommodate these services only in terms of bandwidth and using the conventional aggregation method, which bundles narrow access bandwidths into a large bandwidth, we assume that it will be difficult to realize efficient accommodation because of the wide fluctuation in the access traffic. To meet the above requirement, we are studying a new access network based on multistage semiconductor optical amplifiers (SOAs), that has a multi-access point that accommodates different services separately.

In this paper, we describe the configuration and advantage of this network. Moreover, in consideration of the recent trend to save energy in future networks, we present a traffic design obtained by simulations that examine the number of optical-electrical-optical (OEO) conversions at first-in first-out (FIFO) controlling packets in terms of reducing the power consumption of this network.

\section{Network configuration}

Fig. 1 shows the configuration of the multi-stage access network. This network has multi-access points $(\# 1 \sim \# \mathrm{~N})$, that are aggregated at each service and connected to the network plane $(\# 1 \sim \# \mathrm{~N})$ where such characteristics as the bandwidth and transmission delay are sufficient for each service $(\# 1 \sim \# \mathrm{~N})$.

The optical line terminal (OLT) upstream configuration is shown in Fig. 1 (a). It consists of a checking part with a photo diode (PD) and an electrical buffer and a gating/regenerating part with a fiber delay line (FDL) and a SOA. This delay line setup for timing employs a well-known technique that is used for optical packet switching in photonic networks [3]. And the FDL length depends on the checking packet header time as described later.

In the checking part, all upstream signals are distinguished at each access point by their checking packet header information. If the signals match a service, they are sent to a drop buffer, and the SOA in the gating/regenerating part is $\mathrm{ON} / \mathrm{OFF}$ controlled to prevent the matched signals from passing to the next access point. Drop signals are transmitted on the service plane. In contrast, if the signals do not match a service, the SOA is ON/OFF controlled as the unmatched signals pass to the next access point. The OLT downstream configuration is shown in Fig. 1 (b). Unlike the upstream OLT, it has a checking part with an electrical buffer for adding signals and a gating/regenerating/modulating part with a modulator for downstream signals and adding signals. The procedure for controlling the downstream OLT is described below.

Step1) The checking part checks whether there are any signals in the add buffer. 


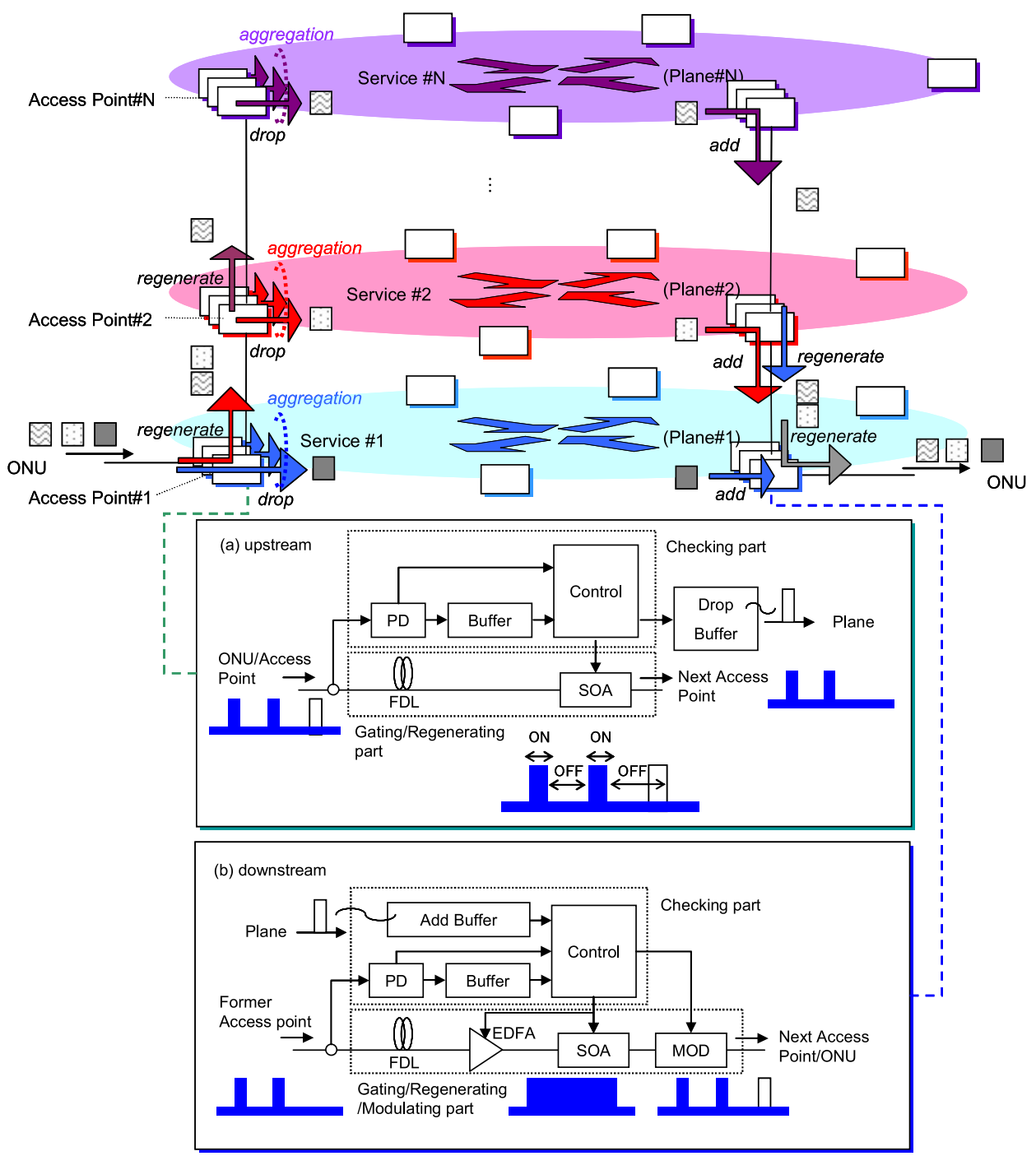

Fig. 1. Configuration of multi-stage access network based on SOA. (a) for upstream, (b) for downstream.

Step2) If signals are present in Step1, any downstream signals are erased by SOA optical saturation using a data rewriting technique [4]. At the same time, the downstream signals are OEO converted and buffered. Then both buffer signals and adding signals are read with FIFO control and modulated. If no signals are present in Step1, the modulation is stopped and all the optical signals are gated or regenerated in the SOA without OEO conversion.

Here an erbium-doped fiber amplifier (EDFA) is only used in the procedure for erasing downstream signals. Therefore, to reduce the power consumption of this network, there should be as few erasing procedures as possible with OEO conversion.

By composing the network as described above, we can design an efficient aggregation for the bandwidth of each service because we can easily calculate the effects of traffic fluctuation on the various services. Moreover, various quality-of-service demands can be met for each service. 
An advantage of this network is that it easily realizes a distribution network that avoids traffic congestion. If service plane\#1 is congested or overflowing, we can use service plane\#2, which handles the same service as plane\#1. On the other hand, if service plane\#1 is not congested or almost free, we can stop using service plane\#2 and thus reduce power consumption. Another merit is that it easily provides the desired delay performance. For example, if service plane\# 1 is composed of a photonic network without OEO conversion, low delay performance is realized free from the influence of any service.

\section{Simulation results}

To investigate ways of reducing the power consumption in a downstream OLT, we estimated the OEO conversion ratio, which we calculated from the ratio of the number of OEO conversions for downstream/adding signals to the total number of downstream/adding signals.

The transmission rate is $1 \mathrm{Gbit} / \mathrm{s}$, which is the sum of the optical signal bandwidth $\left(\mathrm{B}_{\mathrm{opt}}\right)$ and adding signal bandwidth $\left(\mathrm{B}_{\text {add }}\right)$. We assumed that all the optical signals were Ethernet packets, whose frame length was $64 \sim 1518$ bytes (header length $=14$ bytes, data length $=46 \sim 1500$ bytes, frame check sequence $=4$ bytes). The packet header checking time depends on header length. And the waiting time in the buffer and the waiting time in the add buffer can also be calculated by using the frame length and transmission rate. All the packets arrive in accordance with the Poisson process. And the arrival processes of the buffer signals and adding signals are independent.

Fig. 2 (a) shows the OEO conversion ratio of downstream signals $R_{\text {opt }}$ at $\mathrm{B}_{\mathrm{opt}}=100 \sim 500 \mathrm{Mbit} / \mathrm{s}$. We can see that, increases in the optical packet bandwidth and the adding packet bandwidth affect the high OEO conversion. Therefore, to reduce the power consumption in a multi-stage access network, it is important to consider the combination of $\left(\mathrm{B}_{\mathrm{opt}}, \mathrm{B}_{\mathrm{add}}\right)$ and the actual rate of bandwidth utilization at each access point.

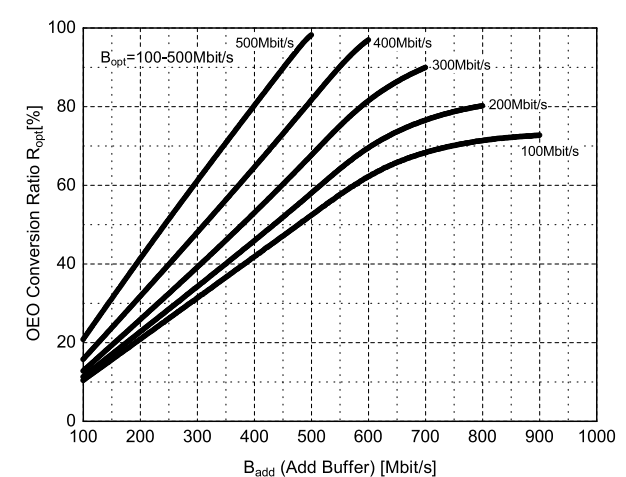

(a)

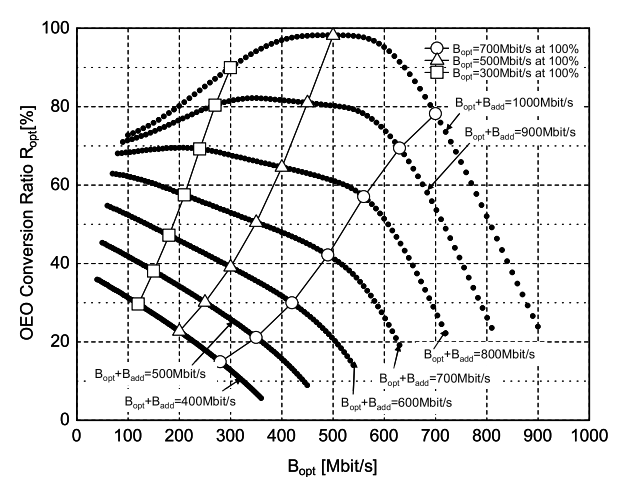

(b)

Fig. 2. (a) OEO conversion ratio at $\mathrm{B}_{\mathrm{opt}}=100$ $-500 \mathrm{Mbit} / \mathrm{s}$. (b) OEO conversion ratio at $\mathrm{B}_{\text {opt }}+\mathrm{B}_{\text {add }}=400-1000 \mathrm{Mbit} / \mathrm{s}$ 
The OEO conversion ratio performance affected by this combination and the bandwidth utilization rate is shown in Fig. 2 (b). Here, we assumed that the actual rate of bandwidth utilization at each access point is $40 \sim 100 \%$ $\left(\mathrm{B}_{\mathrm{opt}}+\mathrm{B}_{\mathrm{add}}=400 \sim 1000 \mathrm{Mbit} / \mathrm{s}\right)$. This corresponds to the day-night traffic variation [5], where the traffic is $100 \%$ at $2: 00$ and $40 \%$ at 14:00. As shown by this graph, at $\mathrm{B}_{\mathrm{opt}}+\mathrm{B}_{\text {add }}=400 \sim 800 \mathrm{Mbit} / \mathrm{s}$, the OEO conversion ratio is the result of a trade off between $B_{\text {opt }}$ and $B_{\text {add }}$. Namely, as the optical signal bandwidth increases, the OEO conversion ratio decreases. However, at $\mathrm{B}_{\mathrm{opt}}+\mathrm{B}_{\text {add }}=900 \sim 1000 \mathrm{Mbit} / \mathrm{s}$, the OEO conversion ratio becomes largest near $\mathrm{B}_{\mathrm{opt}}=\mathrm{B}_{\mathrm{add}}$. Therefore, daily fluctuation in the $\mathrm{OEO}$ conversion increases close to $B_{o p t}=B_{\text {add }}$ if the proportion of $B_{o p t}$ and $B_{\text {add }}$ to time is constant. For example, at $\mathrm{B}_{\mathrm{opt}}=700$ and $300 \mathrm{Mbit} / \mathrm{s}$ at $100 \%$ bandwidth utilization, the fluctuations are $14.9 \sim 78.1 \%$ and $29.9 \sim 89.9 \%$ respectively. Meanwhile, at $\mathrm{B}_{\mathrm{opt}}=500 \mathrm{Mbit} / \mathrm{s}$ at $100 \%$ bandwidth utilization, the fluctuation is $22.7 \sim 98.2 \%$.

In view of these simulation results, we analyzed the multi-access point traffic design to reduce the number of OEO conversions in the entire multistage access network. The evaluation formula is as follows:

$$
N_{\text {oeo }}=\sum R_{\text {opt }}(i) \times B_{o p t}(i)+R_{a d d}(i) \times B_{a d d}(i)
$$

Here, $\mathrm{N}_{\text {oeo }}$ is the evaluation value that corresponds to the total number of OEO conversions. $i$ is the plane number, and $\mathrm{R}_{\text {add }}(=100 \%)$ is the OEO conversion ratio of adding signals.

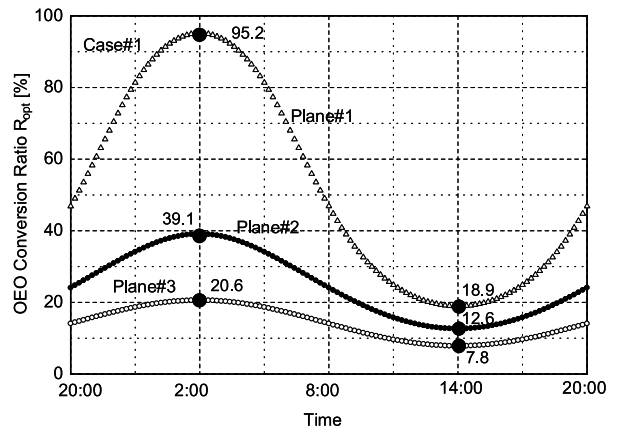

(a)

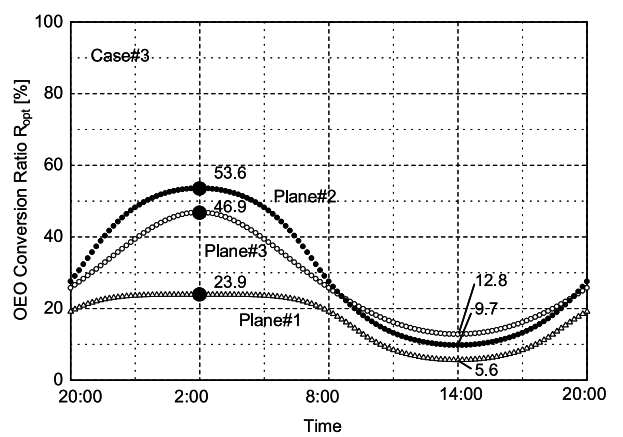

(c)

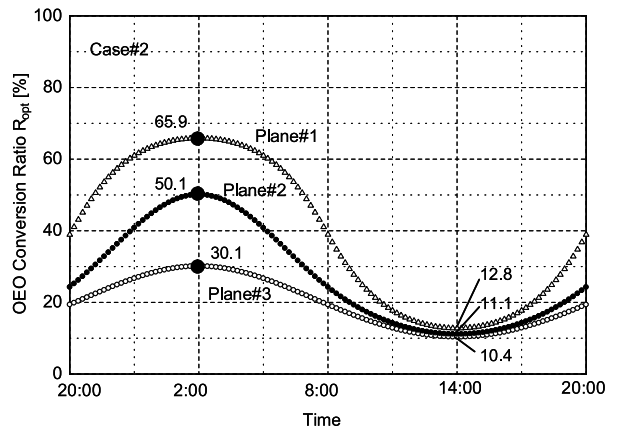

(b)

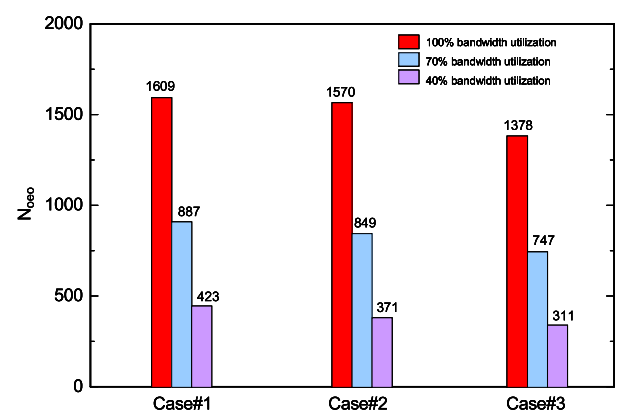

(d)

Fig. 3. OEO conversion ratio and evaluation value in

IEICE 2010

DOI: $10.1587 /$ elex.7.886 Received May 17, 2010

Accepted May 25, 2010 Published June 25, 2010 4-stage access network. (a) is Case\#1, (b) is Case\#2, (C) is Case\#3. (d) is evaluation value in each case. 
Fig. 3 (a)-(c) show the OEO conversion ratio $R_{\text {opt }}$ of each plane in a 4-stage access network. Here, $\mathrm{B}_{\text {add }}[\mathrm{Mbit} / \mathrm{s}]$ (plane\#4, plane\#3, plane\#2, plane\#1) is as follows: Case\#1 $(100,200,300,400)$, Case\#2 $(250,250,250$, 250), and Case\#3 (400, 300, 200,100). A comparison of Fig. 3 (a)-(c) shows that the variation in the OEO conversion ratio $\mathrm{R}_{\text {opt }}$ at $100 \%$ bandwidth utilization (at 2:00) becomes larger in the order given in Fig. $3(\mathrm{c}) \rightarrow$ (b) $\rightarrow$ (a). On the other hand, that at $40 \%$ bandwidth utilization (at 14:00) is approximately the same. Therefore, comparing the $\mathrm{N}_{\text {oeo }}$ values for each case at high bandwidth utilization is especially important.

Fig. 3 (d) shows the $\mathrm{N}_{\text {oeo }}$ of each plane at $40 \%, 70 \%$, and $100 \%$ bandwidth utilization. In terms of reducing the power consumption of the entire multi-stage access network, Case\#3 is the best traffic design of all cases because $\mathrm{N}_{\text {oeo }}$ is the smallest in all bandwidth utilization. Consequently, the bandwidth of the adding signal handled in each plane must be decreased in the ascending order of plane number (bandwidth: $\# \mathrm{~N}>\# \mathrm{~N}-1 \ldots>\# 2>\# 1$ ).

\section{Conclusion}

In this paper, we described the configuration and advantage of a multi-stage access network based on SOA. Moreover, we examined the OEO conversion ratio performance to reduce power consumption. Based on simulation results, to reduce power consumption, the design should be such that the bandwidth of the adding signal handled in each plane decreases in ascending order of plane number. If this traffic design is employed as a multi-stage access network, we can expect to achieve both efficient bandwidth accommodation and low power consumption that will support various services. 